\title{
Perfil do Consumidor de Alimentos Industrializados da Cidade de Guanambi - BA
}

\author{
Processed Food Consumers' Profile in the City of Guanambi - BA
}

\author{
Charlene Neves dos Santos*a; Karoline Fernandes Borges ${ }^{\mathrm{a}}$; Mirelle Costa Pignata-Viana; Denis Harley Nunes Lima ${ }^{\mathrm{a}}$
}

${ }^{a}$ Centro Universitário UniFG. BA, Brasil.

*E-mail: charleneneves@outlook.com

\begin{abstract}
Resumo
O processamento industrial aumenta a vida útil dos alimentos e confere praticidade aos consumidores. O objetivo do trabalho foi avaliar o perfil e a preferência dos consumidores de alimentos industrializados da cidade de Guanambi/BA, durante o período de março a agosto de 2017. Para o estudo foram realizadas entrevistas com 378 pessoas, usando um questionário de múltipla escolha, conforme tamanho da população, homogeneidade do público-alvo e a quantidade de erro amostral admitida (5\%). O consumo de alimentos industrializados foi maior entre o público feminino $(75,1 \%)$, jovem $(33,6 \%)$, e em sua maioria, com $2^{\circ}$ grau completo $(43,7 \%)$ e composição familiar entre dois e quatro membros (78,3\%). A maioria dos respondentes possuía renda entre um e três salários-mínimos (76,3\%). Os consumidores responderam por optar por uma alimentação prática $(49,5 \%)$, com frequência entre três e cinco dias por semana $(32,3 \%)$. Os entrevistados disseram consumir alimentos ricos em gorduras, açúcar e sal, entretanto, $80,7 \%$ dos respondentes consideraram alimentos industrializados não nutritivos, e a maioria (79,4\%) afirmou que deixaria de ingeri-los caso o consumo fosse prejudicial à saúde. Dessa forma, o consumo de alimentos industrializados pode estar associado com fatores culturais, de renda e de disponibilidade de tempos para o preparo das refeições individuais e/ou da família, sendo que o público jovem e feminino apresentou o maior padrão de consumo.
\end{abstract}

Palavras-chave: Hábito Alimentar. Perfil Socioeconômico. Processamento Industrial.

\begin{abstract}
The industrial food processing ncreases shelf life and confers convenience to the consumers. This study aimed to evaluate the profile of processed foods consumers in the city of Guanambi-BA from March to August 2017. 378 consumers were interviewed by choosing a multiplechoice questionnaire taken into account population size, target public homogeneity, and sampling error rate at 5\%. In Guanambi, that profile revealed on its majority women (75.1\%), youngers (33.6\%), with secondary education $(43.7 \%)$, coming from a family size of two to four members (78.3\%). Most interviewees earn one to three minimum wages $(76.3 \%)$. The reason why they choose this kind of food is due to the practicality (49.5\%) with a varying frequency of three to five days a week. Interviewees admit consuming food with a high content of fats, sugar, and salt. However, a little over four-fifths (80.7\%) consider processed food non-nutritive. Nearly $80 \%$ would consider stopping eating them if it were harmful to health. Thus, such consumption may be related to cultural factors, income, and available time to prepare individual and/or family meals, with notably dietary patterns among young and female interviewees.
\end{abstract}

Keywords: Food Habit. Socioeconomic Profile. Industrial Processing.

\section{Introdução}

Alimentos industrializados são aqueles modificados, préprontos ou prontos para o consumo, fabricados utilizando substâncias extraídas de alimentos, como: açúcares, óleos e gorduras, seus derivados constituintes (gorduras hidrogenadas e amido modificado) ou ainda sintetizados em laboratórios (corantes, aromatizantes, conservantes e outros aditivos), e também, a partir de matérias orgânicas. O processamento industrial tem por finalidade prolongar a vida útil dos alimentos, bem como melhorar as características sensoriais e diversificar o consumo de matéria-prima (MOODIE et al., 2013).

O aumento da produção de alimentos industrializados ocorreu em função do rápido e acelerado crescimento populacional e de urbanização, que resultou na expansão do sistema agroalimentar, ampliando assim a necessidade de maior produção, maior qualidade e de mais inovações tecnológicas no processamento de matérias-primas, na melhoria da conservação e distribuição dos alimentos, pois com essa elevação na procura houve a necessidade de controlar, de forma mais rigorosa, o modo como o alimento chegaria ao consumidor, livre de contaminações microbiológicas (CRUZ, 2010).

Durante a fabricação destes alimentos, alguns ingredientes, como: corantes artificiais, espessantes, aromatizantes e conservantes são adicionados, o que pode representar um risco à saúde das pessoas. A indústria também processa alimentos com adição de ingredientes funcionais, tendo como objetivo enriquecer nutricionalmente o produto e, em alguns casos, conferir propriedades funcionais (FERREIRA, et al., 2015).

A indústria alimentícia no Brasil é um ramo em expansão, cuja importância de seu crescimento é atribuída a sua necessidade de produzir alimentos de acordo com o aumento 
da população (CASARIN, 2012). O aumento da renda familiar dos últimos vinte anos permitiu uma mudança no padrão alimentar das pessoas de baixa renda, formando assim um novo mercado de consumo popular, em função de fatores como programas sociais (bolsa família), reajuste do salário mínimo e previdência social (GOUVEIA, 2006).

De acordo com a Associação Brasileira da Indústria de Alimentos, 46,7 bilhões de reais se torna o saldo da balança comercial brasileira dos alimentos industrializados consumidos no Brasil, registrando, assim, 6,7\% de crescimento no rendimento no ano de 2019 (ABIA, 2020a). Diante desses números, houve um faturamento de 699,9 bilhões de reais na indústria de alimentos e bebidas, sendo 250 milhões de toneladas de comida por ano (ABIA, 2020b).

No geral, o hábito e a cultura definem o padrão alimentar em uma determinada região, e esse está fortemente relacionado com a renda familiar e com o preço do produto (MOURA, 2015), sendo cada vez maior o consumo de alimentos industrializados em função de fatores, como: praticidade, rapidez e durabilidade (LEAL, 2010). Dessa forma, conhecer os hábitos alimentares dos indivíduos estabelece o primeiro passo para avaliar seu perfil socioeconômico, para analisar a frequência de consumo desses alimentos e os motivos que levam a população a consumir determinados tipos de produtos.

Diante do exposto, o objetivo do trabalho foi avaliar o perfil e a preferência dos consumidores de alimentos industrializados da cidade de Guanambi/BA.

\section{Desenvolvimento}

\subsection{Metodologia}

O trabalho foi desenvolvido na cidade de Guanambi, localizada na região Sudoeste do Estado da Bahia, durante o período de março a agosto de 2017. Atualmente, a cidade possui 78.833 habitantes, segundo o Instituto Brasileiro de Geografia e Estatística (IBGE) (IBGE, 2010a).

Um questionário estruturado composto por 17 questões de múltipla escolha foi aplicado aos participantes para realizar um levantamento descritivo de informações sobre os hábitos de consumo de alimentos industrializados, destacando o tipo de alimento mais consumido, a frequência de consumo, os motivos que a levam a consumir esses produtos, o conhecimento do valor nutricional e, ainda, algumas informações adicionais como sexo dos/as participantes, faixa etária, grau de escolaridade, renda e composição familiar.

A coleta de dados foi realizada nos turnos matutino e vespertino e as perguntas foram direcionadas apenas para um morador de cada residência, utilizando-se como critério de exclusão os indivíduos que não consumiam esse tipo de produto e apresentavam idade inferior a 18 anos. Aos participantes foi solicitado que lessem e respondessem com atenção, e o máximo de honestidade possível, cada uma das questões. Foram disponibilizados 20 minutos, sendo informados de antemão que avisassem caso achassem necessário dispor de mais tempo.

Informou-se ao participante que caso não quisesse poderia se recusar a responder questões que lhe causassem algum constrangimento, podendo até mesmo desistir de participar da pesquisa, em qualquer momento, sem nenhum prejuízo, ressaltando ainda que sua participação era voluntária, não havendo pagamentos ou cobranças por sua colaboração.

Posteriormente, as informações obtidas com os entrevistados foram analisadas em conjunto com os dados conseguidos com os demais participantes. Os entrevistados foram orientados a ler e assinar o Termo de Consentimento Livre e Esclarecido (TCLE), elaborado de acordo com a Resolução no 466/2012 do Conselho Nacional de Saúde, após aprovação do trabalho pelo Comitê de Ética em Pesquisa do Centro Universitário (UniFG), Guanambi/BA, sob o Parecer $n^{\circ} 864.902 / 2017$.

$\mathrm{O}$ número de consumidores entrevistados foi calculado de acordo com o tamanho da população, homogeneidade do público-alvo (consumidores de alimentos processados), quantidade de erro amostral admitida e grau de confiança de $95 \%$. Por existir na cidade 22.278 domicílios particulares permanentes (IBGE, 2010a), fez-se necessário entrevistar 378 consumidores conforme cálculo da população finita (MIOT, 2011).

As residências foram escolhidas aleatoriamente, entrevistando-se aproximadamente 37 moradores por bairros, em um total de 10 bairros (Vila Nova, Monte Pascoal, Ipanema, Beija Flor I e II, Bela Vista, Santo Antônio, São Francisco, Aeroporto Velho, Vomita Mel e Centro).

Os resultados obtidos foram submetidos à análise de frequência, valendo-se do Programa Excel para isso. Foi realizada uma relação não paramétrica para analisar as correlações entre o consumo de alimentos industrializados e as variáveis de gênero, de renda, de composição familiar, de grau de escolaridade, de faixa etária, de hábitos e de frequência alimentar, bem como o interesse pelo produto e outras variáveis como opinião sobre o produto e motivação para o consumo.

\subsection{Resultados e Discussão}

$\mathrm{O}$ número de residências entrevistadas $(\mathrm{n}=378)$ representou $1,69 \%$ do total de domicílios particulares permanentes, localizadas na cidade de Guanambi (IBGE, 2010a). Dos entrevistados, 75,1\% eram do sexo feminino e $24,9 \%$ do masculino. Essa diferença pode estar relacionada ao ambiente no qual ocorreu a entrevista, sendo esta domiciliar. Quanto à faixa etária se verificou que 33,6\% dos participantes apresentam idades entre 18 e 25 anos e a escolaridade predominante foi do Ensino Médio ( $2^{\circ}$ Grau), com 43,7\% (Quadro 1). 
Quadro 1 - Variável gênero, faixa etária, nível de escolaridade, renda e composição familiar do consumidor de alimentos industrializados da cidade de Guanambi - BA

\begin{tabular}{|c|c|c|}
\hline \begin{tabular}{|l|} 
Variável \\
\end{tabular} & $\mathbf{N}$ & $(\%)$ \\
\hline \multicolumn{3}{|l|}{ Gênero } \\
\hline Masculino & 94 & 24,9 \\
\hline Feminino & 284 & 75,1 \\
\hline \multicolumn{3}{|l|}{ Faixa etária } \\
\hline $18-25$ anos & 127 & 33,6 \\
\hline $26-40$ anos & 123 & 32,5 \\
\hline $41-60$ anos & 108 & 28,6 \\
\hline$>60$ anos & 20 & 5,3 \\
\hline \multicolumn{3}{|l|}{\begin{tabular}{|l|} 
Nível de \\
escolaridade
\end{tabular}} \\
\hline \begin{tabular}{|l|} 
Ensino \\
Fundamental \\
\end{tabular} & 85 & 22,5 \\
\hline Ensino Médio & 165 & 43,7 \\
\hline Ensino Superior & 114 & 30,2 \\
\hline \begin{tabular}{|l|} 
Não alfabetizado \\
\end{tabular} & 14 & 3,7 \\
\hline \multicolumn{3}{|l|}{ Renda familiar $^{1}$} \\
\hline$<01$ salário & 100 & 36,0 \\
\hline \begin{tabular}{|l|} 
Entre 01 e 03 \\
\end{tabular} & 212 & 76,3 \\
\hline \begin{tabular}{|l|} 
Entre 03 e 05 \\
\end{tabular} & 53 & 19,1 \\
\hline Entre 05 e 10 & 13 & 4,7 \\
\hline$>10$ salários & 0 & 0,0 \\
\hline \multicolumn{3}{|l|}{\begin{tabular}{|l|}
$\begin{array}{l}\text { Composição } \\
\text { familiar }^{2}\end{array}$ \\
\end{tabular}} \\
\hline \begin{tabular}{|l|} 
Entre 02 e 04 \\
\end{tabular} & 296 & 78,3 \\
\hline Entre 04 e 06 & 64 & 16,9 \\
\hline$>06$ & 18 & 4,8 \\
\hline
\end{tabular}

${ }^{1}$ Em salário-mínimo; ${ }^{2}$ Número de pessoas.

Fonte: dados da pesquisa.

A ingestão de alimentos processados foi maior entre os indivíduos do sexo feminino $(75,1 \%)$, com maior grau de escolaridade. Bielemann et al. (2015) analisaram o consumo de alimentos ultraprocessados e o impacto na dieta de adultos jovens, com idade média de 23 anos, em pesquisa domiciliar na cidade de Pelotas/RS, e observaram a predominância feminina nos lares visitados, corroborando com a presente pesquisa.

Segundo Avelar (2010), ao realizar uma pesquisa sobre o consumo de alimentos e alimentação fora do lar na cidade de Lavras/MG, concluiu que a idade influenciou na preferência do consumidor quanto à escolha da alimentação, tendo verificado que os indivíduos de idade mais alta são mais conservadores, priorizando a alimentação em casa. $\mathrm{O}$ autor observou também que a partir do grau de escolaridade foi possível delinear o perfil do consumidor, pois auxilia o indivíduo a se posicionar em relação à decisão de compra. Notou-se uma redução da qualidade alimentar entre pessoas com maior nível educacional (superior).

O grau de escolaridade é determinante para uma alimentação saudável e equilibrada (VIEBIG, 2009). No entanto, verificou-se que $30,2 \%$ dos respondentes possuem Ensino Superior, visto que a cidade em que a pesquisa foi realizada é uma cidade universitária, corroborando com a idade predominante no estudo, em sua maioria jovens ( $n=127)$.

Ao realizarem um levantamento sobre a influência da escolaridade na percepção de alimentos considerados saudáveis, Moura et al. (2014) observaram que $42 \%$ dos indivíduos com baixa escolaridade $\left(4^{\circ}\right.$ ano do Ensino Fundamental) demonstraram um conceito equivocado sobre alimentação saudável, pontuando como alimentos nutritivos o suco artificial, biscoito recheado, cereal matinal, maionese, embutidos, macarrão instantâneo e temperos prontos. Tal estudo exemplifica a necessidade da adoção de práticas de educação nutricional no currículo base da educação, sobretudo nas séries iniciais, para o incentivo da tomada de decisão acerca das escolhas alimentares.

Em relação à renda familiar, observou-se que os consumidores entrevistados possuem renda média entre um e três salários-mínimos (76,3\%), enquanto a composição familiar variou entre dois e quatro indivíduos para cada residência, representando 78,3\% dos resultados alcançados (Quadro 1).

Alves et al. (2015), ao avaliarem o perfil do consumidor de leite tipo A, na cidade de Guanambi, no ano de 2012, observaram que $63,7 \%$ dos consumidores apresentaram renda entre um e três salários-mínimos e composição familiar equivalente a $69,4 \%$ (entre dois e três membros da família), resultado inferior ao encontrado no presente trabalho. $\mathrm{O}$ aumento do padrão de renda neste estudo foi considerado baixo em relação ao encontrado pelos autores supracitados, porque não houve crescimento da população para tanto, tendo uma expansão de $2,1 \%$ do faturamento anual, incoerente com o progresso de $8,9 \%$ no número da composição familiar (Quadro 1).

Por agregar valor ao produto, o acesso aos alimentos industrializados por indivíduos de menor nível de renda é mais difícil. Ressaltando-se que existe uma mudança nos hábitos alimentares da população que dispõe de maior poder aquisitivo, como alimentos prontos para o consumo (fast foods) e os doces, tendendo a sofrer mais com problemas de sobrepeso (MORATOYA, 2013). Dessa forma, presume-se que a maior detenção de renda não necessariamente garantirá uma melhor nutrição.

O Instituto Brasileiro de Geografia e Estatística (IBGE, 2010b), por meio do Programa de Orçamento Familiar (POF), ao avaliar a aquisição alimentar domiciliar per capita anual por classes de rendimento total, demonstrou que os padrões de escolha de alimentos processados, como: açúcar, doces, produtos de confeitaria, alimentos preparados e misturas industriais foi maior entre os indivíduos que possuíam renda superior a seis mil reais. Sendo esta uma variável importante no poder de escolha.

A pesquisa realizada pelo IBGE (2010b) revelou ainda que o déficit de peso foi maior entre os indivíduos do sexo masculino (mais de $1 / 4$ até $1 / 2$ salário-mínimo) e feminino (até $1 / 4$ do salário-mínimo) que apresentavam menor poder aquisitivo e à medida que o rendimento aumentou (entre dois e cinco salários-mínimos), observou-se maior excesso de peso e obesidade em ambos os gêneros.

Aquino et al. (2002), ao analisarem o consumo infantil 
de alimentos industrializados e renda familiar, na cidade de São Paulo/SP, perceberam que a ingestão de açúcar foi maior entre as crianças, cujas famílias tinham menor rentabilidade, enquanto que o consumo de achocolatados, chocolates, iogurte, leite em pó modificado e refrigerantes eram mais expressivo entre as crianças que faziam parte de famílias com poder aquisitivo elevado.

Quanto à frequência de consumo de alimentos industrializados, foi verificado que a população não possui o hábito de consumi-los diariamente $(21,4 \%)$, relatando uma maior constância no consumo alimentar $(32,3 \%)$ entre três e cinco vezes por semana. A maior motivação observada pelos consumidores se refere à praticidade $(49,5 \%)$, seguida do fator tempo (30,7\%) e rapidez $(19,8 \%)$, conforme observado no Quadro 2.

Carmo et al. (2015), ao caracterizarem o mercado consumidor de sucos prontos para o consumo, observaram que a praticidade $(70 \%)$ é o fator motivacional na escolha destes alimentos, reforçando o resultado obtido nesta pesquisa. Assis et al. (2004), ao analisarem o consumo de alimentos industrializados na comunidade do centro universitário do Leste de Minas Gerais/MG, observaram que 62,8\% dos entrevistados consomem diariamente óleo de soja e a ingestão de azeite e maionese foi mais expressiva entre os professores respondentes.

Uma pesquisa nacional realizada pela Federação das Indústrias do Estado de São Paulo (FIESP, 2010), sobre o perfil do consumo de alimentos no Brasil, demonstrou que a maioria (34\%) dos brasileiros optam por uma alimentação prática e conveniente, enquanto $23 \%$ preferem alimentos confiáveis e de qualidade nutricional. Assim, verificou-se que a comodidade e a facilidade são as principais prioridades dos brasileiros, pois de maneira geral levam uma vida corrida, reforçando os resultados obtidos no presente estudo quanto à praticidade do consumo de alimentos industrializados (Quadro 2).

Quadro 2 - Frequência e motivação de consumo de alimentos industrializados da cidade de Guanambi - BA

\begin{tabular}{|l|c|c|}
\hline Variável & N & (\%) \\
\hline Frequência (vezes/semana) & & \\
\hline Uma vez & 49 & 13,0 \\
\hline Entre 02 e 03 & 94 & 24,9 \\
\hline Entre 03 e 05 & 120 & 32,3 \\
\hline Diariamente & 81 & 21,4 \\
\hline Quinzenalmente & 34 & 9,0 \\
\hline Motivação & & \\
\hline Tempo & 116 & 30,7 \\
\hline Praticidade & 187 & 49,5 \\
\hline Rapidez & 75 & 19,8 \\
\hline
\end{tabular}

Fonte: Dados da pesquisa.

Em relação ao poder de compra, 29,6\% dos consumidores revelaram escolher o produto pelo sabor, enquanto $22,8 \%$ optaram pela qualidade do alimento processado, seguido da marca $(19,8 \%)$, preço $(16,7 \%)$ e costume $(8,5 \%)$, observandose que a minoria $(2,6 \%)$ levou em consideração a vida útil do produto. Segundo Guerreiro (2006), o sabor foi um dos atributos de maior importância para o alimento, pois esse determina sua aceitação e preferência.

Através dos processamentos industriais, as manufaturas de alimentos criaram estratégias propícias para aumentar o consumo de alimentos ricos em substâncias, que podem causar dependências, agregando ao sabor, açúcar, sódio e gordura, para que o mesmo seja viciante ao paladar dos indivíduos, alcançando uma parte do cérebro que desperta o prazer e provoca o vício alimentar (SAWAYA; FILGUEIRAS, 2013).

Freitas et al. (2015), ao estudarem sobre a interferência das marcas no comportamento de compra do consumidor universitário, observaram que 58,5\% dos respondentes concordaram, parcialmente ou totalmente, com a importância da marca na decisão de compra. Os autores verificaram também que 39,4\% dos discentes preferiram adquirir produtos de uma marca já utilizada, reforçando a importância da marca no processo de compra e consumo.

Quanto ao preço (16,7\%), verificou-se que os entrevistados que afirmaram priorizar esta variável, no momento da compra, foram os mesmos que apresentaram menor poder aquisitivo (36,0\%) (Quadro 1). Uma pesquisa nacional da Federação do Comércio do Estado do Rio de Janeiro revelou que este é o fator mais considerado pelas classes C, D e E no ato da compra de determinado produto (GANDRA, 2015).

Em relação aos tipos de alimentos industrializados consumidos pela população guanambiense se observou que a maioria é proveniente de produtos de laticínios, verificando-se um percentual de 87,0 $(\mathrm{n}=329) ; 74,3(\mathrm{n}=281) ; 71,4(\mathrm{n}=270)$; $64,6(n=238)$ e $58,2 \%(n=220)$ para queijos, iogurte, leite pasteurizado,manteigaerequeijão, respectivamente(Quadro3).

Quadro 3 - Relação dos alimentos industrializados mais consumidos na cidade de Guanambi - BA, 2017

\begin{tabular}{|l|c|c|}
\hline Alimentos Industrializados & N & $\mathbf{( \% )}$ \\
\hline Leite pasteurizado & 270 & 71,4 \\
\hline Iogurte & 281 & 74,3 \\
\hline Macarrão instantâneo & 169 & 44,7 \\
\hline Tempero pronto & 242 & 64,0 \\
\hline Suco artificial & 173 & 45,8 \\
\hline Pizza & 273 & 72,2 \\
\hline Chá em sachê & 63 & 16,7 \\
\hline Cereal matinal & 89 & 23,5 \\
\hline Biscoito recheado & 208 & 55,0 \\
\hline Bolacha & 299 & 79,1 \\
\hline Embutidos & 237 & 79,1 \\
\hline Queijos & 329 & 87,0 \\
\hline Enlatados & 231 & 61,1 \\
\hline Salgados & 248 & 65,6 \\
\hline Doces & 281 & 74,3 \\
\hline Refrigerantes & 231 & 61,1 \\
\hline Margarina & 238 & 63,0 \\
\hline Manteiga & 238 & 63,0 \\
\hline Achocolatado em pó & 229 & 60,6 \\
\hline Salgadinhos & 177 & 46,8 \\
\hline Requeijão & 220 & 58,2 \\
\hline Ketchupe & 229 & 60,6 \\
\hline Maionese & 208 & 55,0 \\
\hline Fast-food & 190 & 50,3 \\
\hline Font Das & & \\
\hline
\end{tabular}

Fonte: Dados da pesquisa. 
O consumo do leite pasteurizado, na cidade de Guanambi, observado por Alves et al. (2015) foi de 34,9\%, inferior ao encontrado nesta pesquisa $(71,4 \%)$. A diferença pode estar relacionada à ingestão de leite cru, apontado pelos autores como o de maior consumo pelos entrevistados. Como este é um produto não industrializado, não foi analisado no presente estudo.

Miranda et al. (2015), ao realizarem uma pesquisa de mercado quanto ao perfil de consumidores de produtos lácteos (iogurte, manteiga e queijo) no município de Contagem/MG, observaram uma maior frequência no consumo de queijo (77\%), seguido do iogurte (72\%) e manteiga (65,33\%), corroborando com os dados obtidos neste estudo em relação à ordem de consumo.

Em dados divulgados pelo Programa Nacional de Saúde, realizado pelo IBGE (2013), verificou-se que entre os marcadores de padrão alimentar não saudável estão o consumo regular de refrigerantes, além de doces, de bolos, de tortas, de chocolates, de balas, de biscoitos ou bolachas doces em cinco dias ou mais na semana, observando-se posteriormente que esse hábito alimentar reduzia com o avanço da idade e aumentava conforme o grau de escolaridade. Esta investigação constatou que $74,3 \%$ dos entrevistados possuem o hábito de consumir doces, enquanto 79,1 e $61,1 \%$ destes afirmaram consumirem bolachas doces e refrigerantes, respectivamente.

De acordo com uma pesquisa realizada pelo Ministério da Saúde, cerca de 30\% dos jovens consomem doces em excesso, cinco vezes ou mais na semana. Tornando o consumo de alimentos ricos em açúcares e gorduras significativos entre os brasileiros, podendo provocar o desenvolvimento de doenças crônicas como diabetes, obesidade e hipertensão (KOPKO, 2016).

Segundo Barros (2016), houve um aumento de 61,8\% de pessoas diagnosticadas com diabetes, 14,2\% com hipertensão e mais da metade da população está acima do peso recomendado, gerando uma transição de desnutrição para obesidade no Brasil, estimando-se um aumento de $60 \%$ em dez anos.

Observou-se que $62,7 \%$ dos respondentes ingeriram embutidos. Estes são amplamente consumidos pela população em função do sabor atrativo e maior vida útil, sendo ricos em sódio e conservantes como os nitritos e nitratos (sais de cura). Tais aditivos são comumente usados para inibir o desenvolvimento do Clostridium botulinum, responsável pelo botulismo alimentar. O consumo excessivo de tais aditivos presentes em carnes processadas, no entanto, proporciona efeitos indesejáveis, uma vez que estes sais reagem no organismo formando as nitrosaminas, compostos com potencial cancerígeno (OMETO, 2015).

Os fast foods, conhecidos como comida rápida, também foram avaliados no estudo. Observou-se que 50,3\% dos guanambienses optam por refeições prontas, representando, assim, um pouco mais da metade da população entrevistada.
O aumento crescente desse tipo de alimentação ocorreu através da mudança no perfil do consumidor, pois esses lanches possibilitam uma alimentação mais rápida e prática, com variação dos ingredientes, marketing bem trabalhado e fabricação em larga escala (AREND; REIS, 2016).

A maioria dos entrevistados $(79,4 \%)$ afirmou que deixaria de consumir alimentos industrializados por questões de saúde, enquanto $8,7 \%$ revelaram que o preço e $6,6 \%$ a mudança de hábito seria a provável desistência do consumo destes produtos. Estes resultados mostraram que mesmo com um baixo grau de escolaridade (Quadro 1), os consumidores têm se preocupado com a saúde, informações estas, na maioria dos casos transmitidas pela mídia, parâmetro que na presente pesquisa $(5,3 \%)$ não interfere nos hábitos do consumidor.

Ressalta-se, ainda, que alguns entrevistados relataram que reduziram ou deixaram de consumir alimentos processados por orientações médicas, por terem desenvolvido algum tipo de doença (como diabetes, hipertensão, obesidade, entre outras). A ingestão frequente de alimentos industrializados pode acarretar ao indivíduo o desenvolvimento de uma sensibilidade em seu organismo ou em seu cérebro, podendo chegar a contrair doenças crônicas degenerativas ou não transmissíveis (BUZZO et al., 2014).

A rotulagem alimentar deve conter todas as características dos alimentos, seus benefícios nutricionais, contribuições e riscos a saúde, todas essas informações devem constar de forma fidedigna, visando auxiliar o consumidor em suas escolhas alimentares (HAWKES, 2006). Quanto ao nível de conhecimento dos consumidores sobre os alimentos industrializados, foi verificado que $51,6 \%$ destes possuem o hábito de ler o rótulo dos alimentos (Quadro 4).

Quadro 4 - Hábitos de consumo e nível de conhecimento dos consumidores de alimentos industrializados da cidade de Guanambi/BA

\begin{tabular}{|l|c|c|}
\hline Variável & N & (\%) \\
\hline Consumo fora do lar & & \\
\hline Sim & 217 & 57,4 \\
\hline Não & 161 & 42,6 \\
\hline Riscos à saúde humana & & \\
\hline Sim & 358 & 94,7 \\
\hline Não & 20 & 5,3 \\
\hline Leitura dos rótulos & & \\
\hline Sim & 195 & 51,6 \\
\hline Não & 183 & 48,4 \\
\hline Uso de temperos prontos & & \\
\hline Sim & 269 & 71,2 \\
\hline Não & 109 & 28,8 \\
\hline Alimentos industrializados nutritivos & & \\
\hline Sim & 73 & 19,3 \\
\hline Não & 305 & 80,7 \\
\hline
\end{tabular}

Fonte: Dados da pesquisa.

O uso de temperos prontos na preparação de alimentos é alto entre os consumidores entrevistados, equivalente a 71,2\% (Quadro 3). Segundo uma pesquisa realizada pelo Instituto 
Brasileiro de Defesa do Consumidor (2015), o brasileiro consome duas vezes mais sódio que o recomendado pela OMS (2,0 g/dia). O estudo traz um alerta aos consumidores para o aumento da ingestão deste componente em alimentos industrializados, demonstrando que condimentos como caldos e temperos prontos possuem, em sua composição, valor médio de 3,15 g/100 g de sódio, ressaltando-se que o consumo em excesso de sal é um dos fatores de risco, que podem gerar doenças crônicas não transmissíveis, como a hipertensão.

O consumo fora do lar tem crescido bastante. Apesar da crise econômica ter afetado o Brasil, muitos brasileiros não mudaram sua rotina nem seus hábitos alimentares. Segundo dados divulgados pelo IBGE (2010b), estima-se que os brasileiros gastam em média $31 \%$ da sua renda com alimentação fora do domicílio. De acordo com a Associação Brasileira das Empresas de Refeições Coletivas (2017), houve uma queda de $0,7 \%$ em relação ao ano de 2016 , em refeições diárias, obtendo um faturamento de $\mathrm{R} \$ 17,3$ milhões de reais. Para Bezerra (2017), a frequência de aquisição de alimentos fora do lar foi de $41,2 \%$.

Foi constatado nesta investigação que $57,4 \%$ dos consumidores adotam a prática de se alimentar fora de casa (Quadro 4). Tal característica se deve à praticidade dos restaurantes self-service e fast foods, já que na maioria das vezes os participantes relataram não possuir tempo suficiente para o preparo das refeições em função de extensa carga horária de trabalho.

Cartocci (2009), ao analisar a produção e a industrialização dos alimentos, afirma que aqueles que passam por procedimentos industriais não são considerados nutritivos, pois durante o processo de industrialização são adicionados alguns ingredientes que mostram como prejudiciais à saúde quando consumidos em excesso. Corroborando com o observado, na pesquisa foi visto que a maioria dos respondentes $(80,7 \%)$ afirmou que esses produtos não possuem valor nutricional elevado. Foi questionado aos participantes se o consumo em excesso de alimentos processados é prejudicial à saúde, e a maioria desses (94,7\%) afirmou que sim (Quadro 4).

De acordo com Monteiro (2009), dietas ricas em carboidratos simples, gorduras saturadas, gorduras trans e sódio, a exemplo de salgadinhos, lanches, produtos de confeitaria e refrigerantes são nutricionalmente desequilibradas e danosas à saúde.

\section{Conclusão}

O consumo de alimentos industrializados, na cidade de Guanambi/Ba, pode ser atribuído aos fatores culturais e às mudanças de hábitos da população, em função da praticidade que estes alimentos oferecem. Fatores socioeconômicos, como renda familiar e escolaridade também influenciam no consumo de alguns alimentos processados.

A discussão sobre o estilo de vida contemporâneo e as escolhas alimentares trazem a importância da discussão sobre políticas educativas e de controle do consumo de alimentos industrializados, frente aos potenciais riscos que estes possuem. Por outro lado, a industrialização é um processo irrevogável, que garante uma maior variedade de alimentos, aumenta seu tempo de vida útil e torna o procedimento mais economicamente viável. O equilíbrio entre tais escolhas é o ponto crucial para a qualidade de vida.

\section{Referências}

ABERC. Associação Brasileira das Empresas de Refeições Coletivas. Mercado real, estimativa de 2017. Disponível em: $<$ http://www.aberc.com.br/mercadoreal.asp?IDMenu=21>. Acesso em: 15 jun. 2020.

ABIA. Associação Brasileira das Indústrias da Alimentação. A alimentação e ciência. 2019. Disponível em:

$<$ https://www.abia.org.br/downloads/aliementacao-e-ciencia. pdf>. Acesso em: 1 jun. 2020.

ABIA. Associação Brasileira das Indústrias da Alimentação. Relatório anual 2019. Disponível em: <https://www.abia.org.br/ downloads/relatorioAnual_2020.pdf $>$. Acesso em:1 jun. 2020.

ALVES, S. L. et al. Perfil e preferência do consumidor de leite cru e tipo "c" no município de Guanambi - BA. Hig. Alim., v.29, p.1187-1192, 2015.

AQUINO, R.C; PHILIPP, S.T. Consumo infantil de alimentos industrializados e renda familiar na cidade de São Paulo. Rev. Saúde Pública, v.36, n.6, p.655-660, 2002. doi: 10.1590/S003489102002000700001

AREND, S. M. F.; REIS, A. M. D. Juventude e restaurantes fast food: a dura face do trabalho flexível. Rev. Katál, v.12, n.2, p.142-151, 2009. doi: 10.1590/S1414-49802009000200003

ASSIS, E. et al. Consumo de alimentos industrializados na comunidade do centro universitário do leste de Minas Gerais. 2004. Disponível em: < https://www.unilestemg.br/revistaonline/ volumes/02/downloads/artigo_15.pdf $>$. Acesso em: 28 jun. 2020.

AVELAR, A. E. S. de. Fatores de influência no consumo de alimentos e alimentação fora do lar. Lavras: Universidade Federal de Lavras, 2010.

BARROS, R. Hábitos dos brasileiros impactam no crescimento da obesidade e aumenta prevalência de diabetes e hipertensão. Ministério da Saúde. 2016. Disponível em: <http://portalarquivos. saude.gov.br/images/pdf/2017/abril/17/Vigitel_17-417final.pdf $>$. Acesso em: 9 jun. 2020.

BEZERRA, I.N. et al. Consumo de alimentos fora do lar no Brasil segundo locais de aquisição. Rev. Saúde Pública, v.51, n.15, p.18, 2017. doi: 10.1590/S1518-8787.2017051006750

BIELEMANN, R. M, et al. Consumo de alimentos ultraprocessados e impacto na dieta de adultos jovens. Rev. Saúde Pública, v.49, n.28, p.1-10, 2105. doi: http://dx.doi.org/10.1590/ S0034-8910.2015049005572

BRASIL. Ministério da saúde. Agência Nacional de Vigilância Sanitária. Resolução RDC nº 360, de 23 de dezembro de 2003. Aprova o Regulamento Técnico sobre Rotulagem Nutricional de Alimentos Embalados. Diário Oficial [da] República Federativa do Brasil, Brasília, 2003.

BUZZO, M. L. et al. Elevados teores de sódio em alimentos industrializados consumidos pela população brasileira. Rev. Inst, Adolfo Lutz, v.73, n.1, p.32-39. 2014. doi: 10.18241/007398552014731587

CARMO, M.C.L.; DANTAS, M.I.S.; RIBEIRO, S.M.R. Caracterização do mercado consumidor de sucos prontos para o 
consumo. Braz. J. Food Technol., v.17, n.4, p.305-309, 2014. doi: 10.1590/1981-6723.2914

CARTOCCI, C.M. Produção e industrialização de alimentos. Brasília: Universidade de Brasília. 2009.

CASARIN, V. Produção de alimentos: o desafio do século. Inform. Agronôm., n.139, p.24, 2012.

CRUZ, F.T.; SCHNEIDER, S. Qualidade dos alimentos, escalas de produção e valorização de produtos tradicionais. Rev. Bras. Agroecol., v.5 n.2, p. 22-38, 2010.

FERREIRA, J.S.G. et al. Marketing de alimentos industrializados destinados ao público infantil na perspectiva da rotulagem. Vigilância Sanitária Debate, v.3, n.3, p75-84, 2015. doi: 10.3395/2317-269x.00293

FIESP. Federação das Indústrias do Estado de São Paulo. Pesquisa nacional Fiesp/IBOPE sobre o perfil do consumo de alimentos no Brasil. 2010. Disponível em: <http://www.abic.com.br/media/ EST_PESQFoodTrendsl.pdf>. Acesso em: 12 jul. 2020.

FREITAS, R.A. et al. O poder das marcas: um estudo sobre a influência da marca no comportamento de compra do consumidor universitário. Viçosa: Universidade Federal de Viçosa, 2015.

GANDRA, A. Preços determinam decisão de compra dos consumidores, indica pesquisa. Rio de Janeiro, Agência Brasil, 2015. Disponível em: <http://agenciabrasil.ebc.com.br/economia/ noticia/2015-03/precos-determinam-decisoes-de-compra-dosconsumidores-brasileiros-indica>. Acesso em: 20 jun. 2020.

GOUVEIA, F. Indústria de alimentos: no caminho da inovação e de novos produtos. Inovação Uniemp, v.2, n.5, p.32-37, 2006.

GUERREIRO, L. Dossiê Técnico: Panificação. Serviço Brasileiro de Respostas Técnicas (SBRT) 2006. Disponível em: <http:// www.respostatecnica.org.br/dossie-tecnico/downloadsDT/ $\mathrm{Mjc}=>$. Acesso em: 26 jun. 2020.

HAWKES, C. Informação nutricional e alegações de saúde: o cenário global das regulamentações/ Organização Mundial da Saúde. Brasília: Organização Pan-Americana da Saúde; Agência Nacional de Vigilância Sanitária. 2006.

IBGE. Instituto Brasileiro de Geografia e Estatística. Domicílios permanentes, particulares. 2010a. Disponível em:

$<$ http://www.ibge.gov.br/cidadesat/topwindow.htm?1>. Acesso em: 27 jun. 2020.

IBGE. Instituto Brasileiro de Geografia e Estatística. Pesquisa de Orçamentos Familiares (POF). Aquisição alimentar domiciliar per capita, Brasil e grandes regiões. 2010b. Disponível em: $<$ http://biblioteca.ibge.gov.br/visualizacao/livros/liv47307.pdf $>$. Acesso em: 25 jun. 2020.

IBGE. Instituto Brasileiro de Geografia e Estatística. Pesquisa Nacional de Saúde 2013. Percepção do estado de saúde, estilos de vida e doenças crônicas. Brasil, Grandes regiões e Unidades de Federação. 2014. Disponível em:
<ftp://ftp.ibge.gov.br/PNS/2013/pns2013.pdf>. Acesso em: 26 jun. 2020.

IDEC. Instituto Brasileiro de Defesa do Consumidor. Idec faz alerta: brasileiros consomem sódio de mais. 2015. Disponível em: $\quad<$ https://idec.org.br/o-idec/sala-de-imprensa/release/idecfaz-alerta-brasileiros-consomem-sodio-demais $>$. Acesso em: 20 jun. 2020.

KOPKO, G. Cerca de 30\% dos jovens consomem doces em excesso. Portal Da Saúde. 2016. Disponível em:

$<$ http://portalsaude.saude.gov.br/index.php/cidadao/principal/ agencia-saude/23027-cerca-de-30-dos-jovens-consomem-docesem-excesso>. Acesso em: 9 jun.2017.

LEAL, D. Crescimento da alimentação fora do domicílio. Segurança Alim. Nutr. v.17, n.1, p.123-132, 2010. doi: 10.20396/ san.v17i1.8634806

MIOT, H.A. Tamanho da amostra em estudos clínicos e experimentais. J. Vasc. Bras., v.10, n.4, p. 275-278, 2011. doi: 10.1590/S1677-54492011000400001

MIRANDA, M. P. C. et al. Pesquisa de mercado: perfil de consumidores de produtos lácteos (iogurte, manteiga e queijo) no município de Contagem - MG. In: CONGRESSO BRASILEIRO DE MEDICINA VETERINÁRIA; CONGRESSO SULBRASILEIRO DA ANCLIVEPA. Curitiba, p.1585-1589, 2015.

MONTEIRO, C. A. Nutrition and health. The issue is not food, nor nutrients, so much as processing. Public Health Nutr., v.12, n.5, p.729-731, 2009. doi: 10.1017/S1368980009005291

MOODIE, R. et al. Profits and pandemics: prevention of harmful effects of tobacco, alcohol, and ultra-processed food and drink industries. Lancet, v.12, p.1-10, 2013. doi: 10.1016/S01406736(12)62089-3

MORATOYA, E. E. et al. Mudanças no padrão de consumo alimentar no Brasil e no mundo. Rev. Politica Agrícola, n.1, p.7284, 2013.

MOURA, A.F.; MASQUIO, D.C.L. A influência da escolaridade na percepção sobre alimentos considerados saudáveis. Rev. Educ. Pop, v.13, n.1, p.82-94, 2014. doi: 10.14393/REP-v13n12014$\operatorname{art07}$

MOURA, N.C. Influência da mídia no comportamento alimentar de crianças e adolescentes. Segurança Alim. Nutr., v.17, n.1, p. 113-122, 2015. doi: 10.20396/san.v17i1.8634805

SAWAYA, A.L; FILGUEIRAS, A. "Abra a felicidade"? Implicações para o vício alimentar. Estudos Avançados, v.27, n.78, 2013. doi: 10.1590/S0103-40142013000200005

VIEBIG, R.F. et al. Consumo de frutas e hortaliças por idosos de baixa renda na cidade de São Paulo. Rev. Saúde Públ., v.43, n.5, p.806-813, 2009. doi: 10.1590/S0034-89102009005000048

WHO. World Health Organization. IARC Monographs evaluate consumption of red meat and processed meat. Int. Agency Res. Cancer; 2015. 